\title{
Impact of conflict resolution parameters on combined alternate-directions lane assignment and reservation-based intersection control
}

\author{
Aleksandar Stevanovic ${ }^{1 *}$ (D) and Nikola Mitrovic ${ }^{2}$ (D)
}

\begin{abstract}
We recently proposed a concept, called Combined Alternate-Direction Lane Assignment and Reservation-based Intersection Control (CADLARIC), for organizing directionally unrestricted traffic flows in automated vehicle environment. The conflicts between through movements are handled by a reservation-based algorithm while the turning conflicts at the intersections are avoided altogether. This paper extends this research by analyzing the impacts that CADLARIC's parameters, used to control the conflict-resolution processes, have on the efficiency and surrogate safety indicators. The investigated parameters include: (i) buffer time in cell's reservation schedule; (ii) allowed speed to cross the reserved cell; (iii) distance from intersection from which a vehicle can make reservation, and (iv) duration of the lane-change process. For most of the investigated parameters, the numerical results show that less efficient operations lead not just to an increase in delay time and number of stops but also increase number of conflicting situations, because of vehicular queues formed within the intersection zone.
\end{abstract}

Keywords: Innovative traffic organization, Automated intersection control, Autonomous and connected vehicles, Reservation-based intersection control

\section{Introduction}

The current methods of organizing traffic flows in urban networks use directional right-of-way road segments (links) to move traffic between urban intersections. Resolution of conflicts between vehicles is almost exclusively reserved for road intersections, which turns these facilities into conflict "hot-spots" which, when traffic demand exceeds their capacities, can create bottlenecks of our urban traffic networks. This restriction of how traffic is organized is not limited only to the field conditions. Even an attempt to model a different organization of traffic in urban networks hits a major barrier, because the traditional simulation modeling tools do not offer enough

\footnotetext{
* Correspondence: stevanovic@pitt.edu; http://orcid.org/0000-0003-109 3340;

'Department of Civil \& Environmental Engineering, Swanson School of Engineering, University of Pittsburgh, 218D Benedum Hall, 3700 O'Hara Street, Pittsburgh, PA 15261, USA

Full list of author information is available at the end of the article
}

flexibility to model bidirectional traffic on individual road segments.

To address this issue and investigate how a flexible road and traffic organization can impact efficiency of urban networks, the authors developed FAUSIM (Flexible Arterial Utilization SImulation Model) - a novel, agent-based, microsimulation platform. The FAUSIM enables modeling of flexible utilization of urban roads (Stevanovic and Mitrovic [20]) and its outputs have been validated, successfully, through a comparison with a commonly utilized Vissim model (Stevanovic and Mitrovic [21]). Previous experiments of the same researchers illustrated the ability of FAUSIM to model conventional and unconventional traffic control scenarios (Stevanovic and Mitrovic [21]). For example, FAUSIM was used to model a CADLARIC scenario where directional driving paths are altered between neighboring lanes. When turning traffic is then aligned to proper lanes, the vehicles turn (left and right) at intersections (if enough number of lanes is available) without facing any conflicts with 
the other vehicles. Through vehicles are still required to reserve their time-space slots and avoid conflicts with the other through vehicles, as in common reservationbased algorithms (Dresner and Stone [5], Dresner and Stone [6]).

In addition to CADLARIC, FAUSIM can be used to model conventional fixed-time (FT) control, intelligent intersection management scenarios, and others. The initial results of the experiments with CADLARIC, executed on a small three-intersection corridor, have shown that such flexible-arterial-utilization concepts significantly outperform conventional driving with the FT control (Stevanovic and Mitrovic [21]). The benefits of CADLARIC were observed both from surrogate safety and efficiency perspectives. However, it is understandable that more safety-conservative scenarios increase delays, and conversely efficiency is improved when hard surrogate-safety constrains are relaxed. It is important to note here that resolution of vehicular conflicts has been used as a proxy of a conflict between two vehicles, which itself is only a surrogate safety indicator.

This trade-off between efficiency and safety warrants further investigation, especially from the perspective of how various parameters (which are used to organize traffic and resolve conflicts in similar traffic organization schemes) can impact some basic efficiency and surrogate-safety measures. Considering that CADLARIC is an original and very new concept there has not been any previous attempts to perform such investigation.

This paper takes experiments with CADLARIC to the next level by addressing this trade-off between surrogate-safety and efficiency through an analysis of parameters used to control the conflict-resolution processes applied in the CADLARIC (and similar reservation-based urban traffic control scenarios). Thus, the goal of the paper is to investigate and document how various conflict-resolution parameters, deployed in the CADLARIC, as a representative of a novel intelligent urban control scenarios, impact a number of safety and efficiency performance measures.

In the next section we first cover a brief literature review of the most relevant state-of-the art studies. In the following, methodological, section we describe briefly the most important conflict-resolution components of the CADLARIC and we identify the most important parameters which impact various conflict resolution procedures. The following sections covers results of the sensitivity analysis identifying points/regions of "profitable trade-off points" where neither too much efficiency is lost nor too much safety is sacrificed to obtain a decent solution. This section is followed by concluding remarks, which summarize findings and provide directions for future research.

\section{Literature review}

The existing research on novel concepts for urban traffic management and control related to Reservation-Based Intersection Control (RIC) in a Connected and Automated Vehicle (CAV) environment has started about a decade and a half ago. Some of the early studies on this subject include the research on AVs to form flexible platoons using a dedicated short-range communication (Kato et al. [10]). The first notable study on RIC was done by Dresner and Stone (Dresner and Stone [4], Dresner and Stone [5], Dresner and Stone [6]). This seminal work has been followed up by a number of studies that address similar concepts of RIC, often also called automated intersection control or intersection management (Lee and Park [11], Wu et al. [28], Li et al. [15], Ahmane et al. [1], Hassan and Rakha [7], Lin et al. [16], Tian et al. [23], Shahriari and Biglarbegian [18], Levin and Boyles [12], Levin et al. [14], Sun et al. [22], Yang et al. [29]). Each of this studies is original on its own way (mostly by introducing a different technique to solve the scheduling problem), but most of them are similar in an attempt to integrate trajectory design into the signal control (Lee and Park [11], Wu et al. [28], Li et al. [15], Ahmane et al. [1], Hassan and Rakha [7], Lin et al. [16], Sun et al. [22], Yang et al. [29]).

The other group of studies includes those that address space-sharing coordination between moving units, with the objective to enable their safe and efficient movement (Tian et al. [23], Shahriari and Biglarbegian [18], Altche et al. [2], Vu et al. [25], Hausknecht et al. [8], Choy et al. [3], Lee and Park [11]). Some of the most recent studies combine RIC with other concepts such as dynamic traffic assignment, and discuss some of the paradoxes that are observed when RIC is coupled with the other network dynamics (Levin and Boyles [12], Levin et al. [14]). Although most of the aforementioned studies bring very significant contributions to this emerging field of urban traffic operations, none of them addresses a potential challenge to fully utilize road infrastructure without strong directional constraints.

A study of Levin and Boyles [13] probably comes closest to the concept presented in this paper. However, it considers a dynamic lane reversal, where the number of lanes for each direction can be changed dynamically based on the traffic conditions, but never fully separated in a lane-directional traffic fashion. The concept analyzed in CADLARIC case goes a step further by splitting a directional traffic into individual lanes (based on the desired movements at the downstream intersection) and combining it with the RIC. Consequently, the experiments presented in this paper are the first attempt to analyze impact of the most critical parameters for CADLARIC operations, on the efficiency and surrogate safety indicators of traffic in urban networks. 


\section{Methodology}

In few words, instead of using principles of "common" reservation-based intersection management systems (Dresner and Stone [5], Dresner and Stone [6]) where all of the vehicular conflicts are handled at intersections, the proposed CADLARIC approach first assigns various turning flows to different lanes in an alternate fashion, as depicted in Fig. 1. In this way, all of the left- and right-turning movements go through an intersection without any crossing conflicts, reducing potential intersection conflicts only to those between through-moving vehicles (see colored cells in the center of intersection). While we test this approach in road capacity-reach environment (3 lanes in each direction of all approaches) the CADLARIC concept is expected to bring some benefits even in more capacity-restricted environments, which will be focus of our future research. Also, it should be noted that at this point CADLARIC assumes a fleet $100 \%$ consisting of the CAVs and there is no interaction (and inclusion) of pedestrians, bicyclists, and other multimodal transportation system users.

A Reservation-based Intersection Control (RIC) system, based on first-come-first-serve (FCFS) rule and similar to one presented in Dresner and Stone [5] and Dresner and Stone [6], is used to handle conflict avoidance, between through-moving vehicles, at the intersection itself. The RIC system or often called "automated intersection management" (AIM) reserves the necessary cells for all through-moving vehicles approaching the intersection. For instance, for eastbound vehicles the AIM needs to reserve cells with red color (full and stripes) shown in Fig. 1 whereas for the north-bound vehicles the solid colors (red and yellow) cells need to be reserved (see Fig. 1). The cell used for both east-west and north-bound vehicles is red solid cell. Before a vehicle sends the reservation request to the AIM it has to satisfy each of the following three conditions:

- Condition 1: Vehicle travels in the desired lane; i.e. a vehicle has already adjusted its position to the lane dedicated to vehicle's movement at downstream intersection. One should note here that vehicles cannot change lanes within the intersection box and the segment between intersections is the only facility where lane-changing is permitted. Also, it is important to note that for the proposed concept each alternate lane is used by traffic from an opposite direction (Fig. 1). This means that, if a vehicle wants to go in another lane of the same direction, it needs to change a lane twice (see Fig. 1). The lanechanging maneuvers in CADLARIC are often

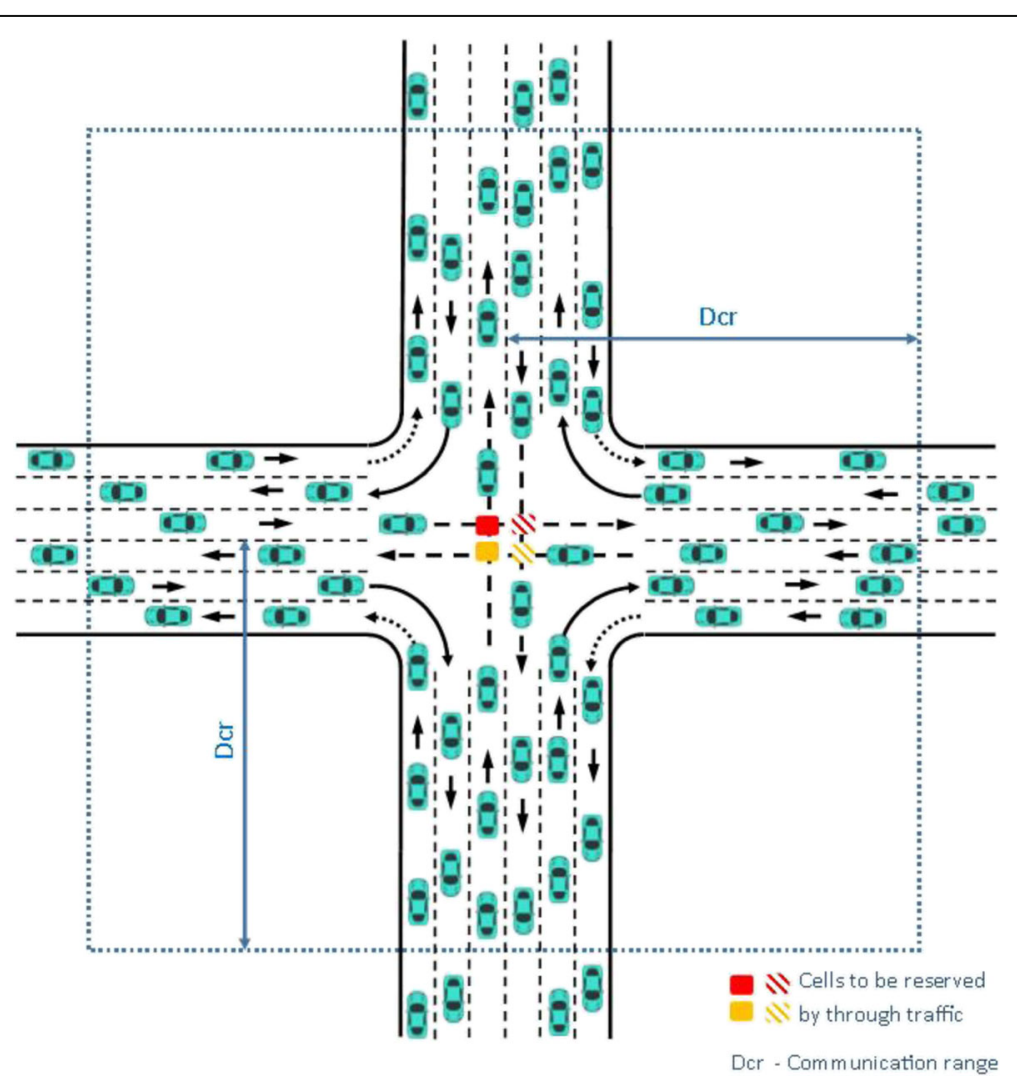

Fig. 1 Lane allocation in CADLARIC control scheme 
executed in a cooperative way where the key input parameter for the control of lane-changing operations is duration of the lane-changing maneuver (see $\tau$ in Fig. 2). For a detailed explanation of the lanechanging algorithm, and its validation through a comparison with Vissim, we refer reader to other publications (Stevanovic and Mitrovic [20]). Furthermore, for a detailed explanation on the resolution of lane-changing conflicts within CALDARIC, we refer reader to a recent publication from the same authors [17]. In this paper, we investigate how various duration of lane changing maneuvers, often set from $1 \mathrm{~s}$ - 3 s (Toledo and Zohar [24]), impact surrogate safety and efficiency indicators of the CADLARIC control strategy.

- Condition 2: Vehicle is within the predefined distance from the intersection. Only if within such a distance from the intersection (see blue box in Fig. 1), a vehicle can establish communication with the Automated Intersection Manager (AIM) and reserve a set of space blocks, which may be also requested by the other vehicles. We examine how the maximum communication range distance $\left(\mathrm{d}_{\mathrm{cr}}\right)$ impacts surrogate safety and efficiency indicators of the proposed CADLARIC control strategy.

- Condition 3: The leading vehicle (if any) has already reserved the corresponding space blocks. This requirement guarantees that the FCFS principle is satisfied and allows the following (or investigated) vehicle to make a reservation.

Once a vehicle fulfills all three conditions it will attempt to reserve a space-time block within the downstream intersection. A corresponding reservation request to the AIM will include parameters such as time of arrival, velocity of arrival at the intersection, etc. (Dresner and Stone [6]). The AIM will review provided information and examine whether or not it will be safe for the vehicle to cross the intersection. If the requested movement through the intersection is unsafe then the AIM repeats the same examination procedure for a $\Delta \mathrm{T}$ postponed arrival time and a corresponding (lower) velocity of arrival (Dresner and Stone [6], Stevanovic and Mitrovic [21]). It is important to note that once within an intersection box vehicles cannot change their traveling speed; i.e. acceleration/deceleration is allowed only while vehicles traverse links between intersections (Dresner and Stone [6], Stevanovic and Mitrovic [21]). The vehicle takes $t_{i n t}[\mathrm{~s}]$ to traverse each cell within the intersection box, with the constant speed of $v_{i n t}$ where $t_{i n t}=\left(\mathrm{l}_{\mathrm{veh}}+\right.$ $\left.\mathrm{l}_{\text {cell }}\right) / v_{\text {int }} . \mathrm{l}_{\mathrm{veh}}$ and $\mathrm{l}_{\text {cell }}$ are lengths of the vehicle and a cell, respectively.

For each of the reserve-able cells assigned to the vehicle path, the AIM attempts to satisfy the vehicle's request in the reservation schedule according to an intersection control policy. An example of the reservation schedule is given in Fig. 3 and it shows, for instance, that the cell $k$ is assigned to the vehicle $i$ at the time $j$.

The intersection control policy, defines, among other parameters, the minimum amount of time that a cell should remain unoccupied between any two consecutive reservations. We refer to this as a buffer time in cells reservation and denote it with $\alpha$ (see Fig. 3). It is expected to see that a larger value of $\alpha$ would lead to "safer operations", while a lower $\alpha$-s would result in more efficient operations (e.g., in terms of delay time and stops). We run multiple scenarios, by changing $\alpha$, to evaluate such impacts of $\alpha$ on efficiency and surrogate safety performance measures.

The intersection control policy also defines the maximum amount of time during which a single cell can be occupied by one vehicle (see $\beta$ in Fig. 3). This is achieved by assigning a speed of arrival $v_{\text {int }}$ to be at least equal to the (predefined) minimum speed for traversing the reserved cells $v_{\beta}$. It is important to note that if the AIM cannot reserve the requested cells, then the approaching vehicle have enough time to stop before the downstream intersection (and wait for the allocated time-space blocks) (Stevanovic and

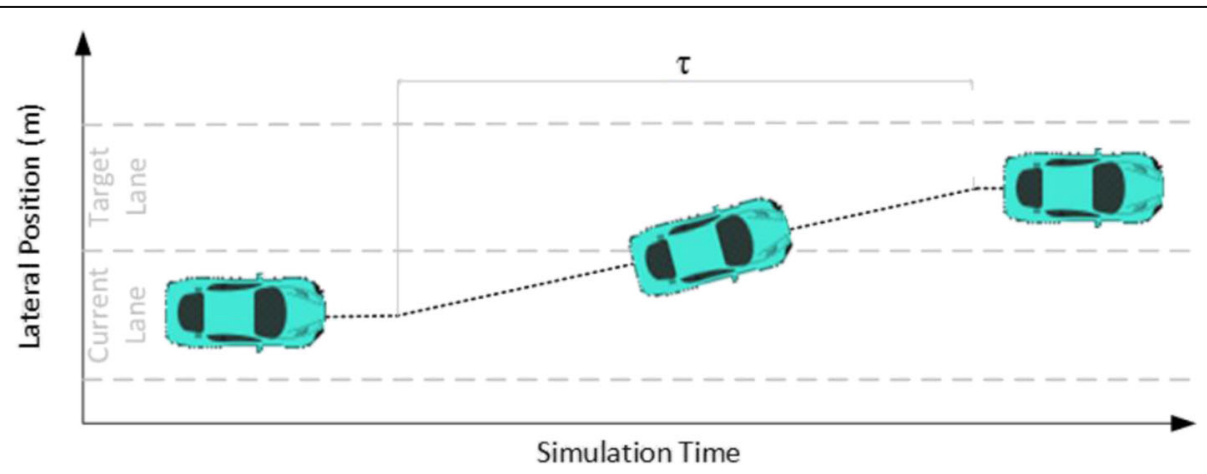

Fig. 2 Lane-changing process 


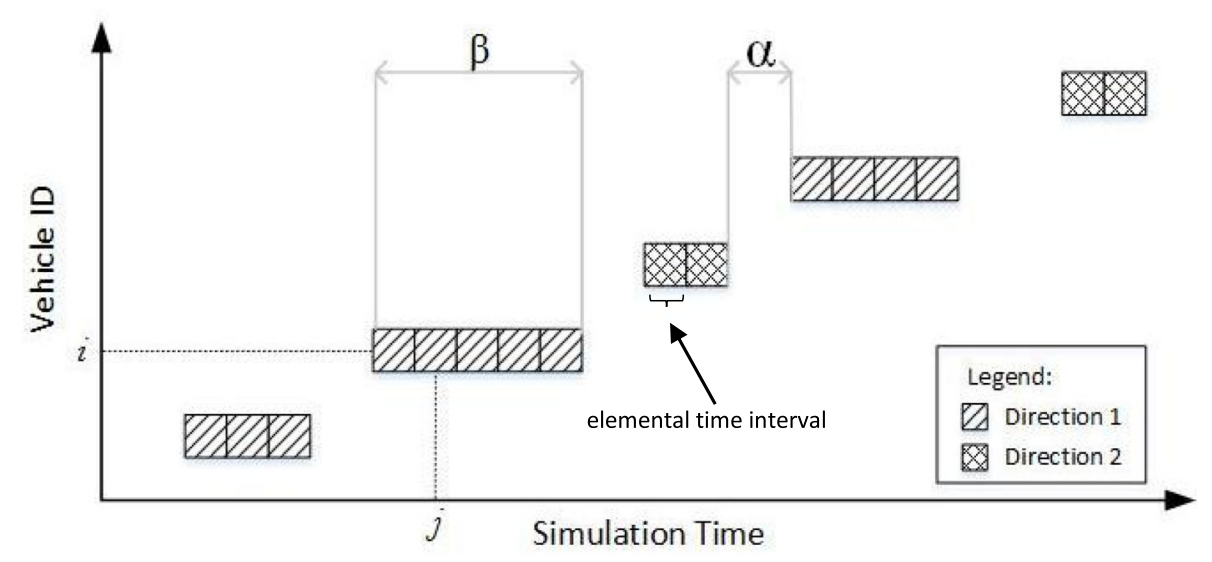

Fig. 3 Reservation schedule of cell k

Mitrovic [21]). Noteworthy, since the vehicle enters and traverses the intersection with the speed $v_{i n t}$ (where $v_{i n t} \geq v_{\beta}$ ), a certain space, in front of the intersection's stop line, needs to be allocated so that a stopped vehicle can accelerate and reach the assigned speed before entering the intersection box. In this way, it is ensured that available time-space blocks within the intersection box are utilized in the most efficient manner.

\section{Experimental setup}

\subsection{Implementation framework}

To model the proposed CADLARIC concept, i.e. a flexible utilization of the road infrastructure, it is first necessary to create a modeling environment that can allow such flexibility, given that most of the available commercial tools (e.g., Vissim, Aimsun) and opensource platforms (e.g., SUMO) do not offer such option. For this purpose, we have developed a new simulation environment, called Flexible Arterial Utilization SImulation Modeling (FAUSIM) (Stevanovic and Mitrovic [21]). FAUSIM is developed within NetLogo modeling platform (developed by Wilensky [27]), through a series of custom-made codes written in Scala, a java-compatible language. Similarly to Cellular Automata model (developed by WageningenKessels et al. [26]), NetLogo platform discretizes space into cells while visualizing simulation entities. To overcome shortcomings of the cell-based traffic models (and deploy more advanced car-following procedures), we have converted the discrete space within NetLogo into a continuous space by relaxing vehicle's parameters (e.g., speed, position) to non-integer values (Wilensky [27]). For more details on how FAUSIM works a reader is encouraged to find relevant information elsewhere (Stevanovic and Mitrovic [21]).

\subsection{Test network}

All of the experiments are performed on a 3-intersection network shown in Fig. 4. Traffic flows (in [veh/hr]) are given, next to the capital letters, for each turning movement. For the sake of simplicity and initial evaluations, the investigated traffic flows consist only of private cars. The speed limit is set to $50 \mathrm{~km} / \mathrm{h}$ and the vehicle's desired speed is uniformly distributed around the speed limit (i.e. within $5 \mathrm{~km} / \mathrm{h}$ on each side of speed limit) It is assumed that all CAVs will strictly follow their randomly assigned desired speeds. OD points are denoted with the encircled numbers next to each of the network entrances/exits (see Fig. 4). Lane assignments at the intersections are set as displayed in Fig. 1. Each movement is also associated with a Level of Service (LOS) [9], represented by the capital letters, where a LOS represents prevailing traffic congestion, for a corresponding optimal fixed-time signal timing plan (Stevanovic and Mitrovic [21]). The length of the considered corridor is $560 \mathrm{~m}$ while the distance between centers of any two adjacent intersections is $200 \mathrm{~m}$. All of the experiments were done with a fleet consisting of $100 \%$ CAVs and no other CAV penetration rates were considered.

\subsection{Evaluated scenarios}

In the developed framework a vehicle $i(\mathrm{i} \in \mathcal{L}$, where $\mathcal{L}$ is the associated volume input) enters the network at time $t$ at one of the entering nodes $(E N\{1, . .8\})$ and with assigned desired speed $v_{i}$. At the entering link, the vehicle is assigned to the lane $l_{j}(j=\{1,2 . .6\})$ dedicated for the movement that vehicle $i$ intends to make at the downstream intersection $z_{j}$ $(j=\{1,2,3\})$. To safely cross the intersection the vehicle $i$ must inform the 'intersection manager' about its attention and request a set of reserve-able the cells $S_{j}$ at downstream intersection (see Dresner and Stone [4] and Dresner and Stone [6]). Right- and left- turning vehicles are exempted from the reservation requests (since they have no conflicts, 


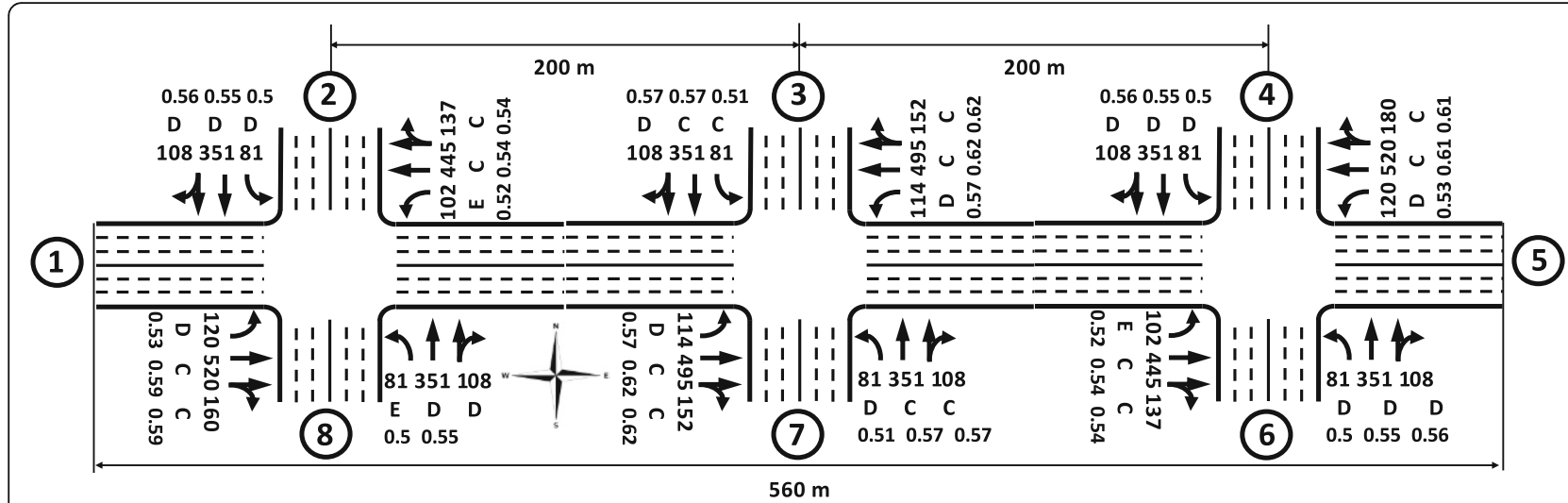

Fig. 4 Geometric and traffic characteristics of the test network

at intersections, with other vehicles) which makes the proposed framework quite unique [17].

Table 1 lists the parameters analyzed in this study and their numerical values used in various simulation experiments. In the first set of experiments, we explored how the reservation buffer time, i.e., time gap between two consecutive arrivals of vehicles at the reserved cell (either from the same or conflicting movements) impacts the efficiency performance measures and surrogate safety indicators. In the second set of experiments, we investigated how the minimum speed for traversing the reserved cells impacts performance of the CADLARIC control strategy. In the third set of scenarios we analyzed the impact of the communication range distance on the efficiency performance measures. Finally, in the forth scenario we explored how the duration of lane changes impacts the number of the corresponding conflicts.

During each simulation experiment only a single corresponding parameter was altered while all of the others were kept constant, at their nominal values that are shown in bold in Table 1. For instance, the minimum allowed speed of through moving vehicles across the reserved cells is set to $20 \mathrm{~km} / \mathrm{h}$ for scenarios 1,3 , and 4 . Five random seed simulation repetitions, each leading to identical vehicle arrivals for all of the experiments in the network, are used to mimic variability of traffic demand for these experiments. In total, we performed 85 simulations, where the total number of simulated vehicles was between 4831 and 4845 .

\subsection{Efficiency performance measures}

Two types of indicators were used to assess the performance of the described control schemes in terms of the traffic efficiency: total delay time and total number of stops. For any given vehicle $i$, the delay is computed as a difference between the actual travel time $\tau_{\mathrm{i}}^{\text {act }}$ for vehicle $i$ to travel from its origin to the destination and the free-flow travel time $\tau_{i}^{f f}$. The total delay in the network is then calculated as:

$$
T D=\sum_{i \in \mathcal{L}}\left(\mathrm{\tau}_{i}^{a c t}-\mathrm{\tau}_{i}^{f f}\right)
$$

where $\mathcal{L}$ refers to the set that contains all vehicles in simulation.

The total number of stops is given by Equation 2 where $\mathcal{N}_{i}$ represents the number of stops vehicle $i$ experiences while traveling from its origin to the destination. Note that a stop is defined as an event when a vehicle's speed drops below a threshold value (e.g., $8 \mathrm{~km} / \mathrm{h}$ ).

$$
T S=\sum_{i \in \mathcal{L}} \mathcal{N}_{i}
$$

\subsection{Conflicting performance measures}

For the sake of assessing impacts of the aforementioned control schemes on conflict resolutions between vehicles, we used two types of performance measures: number of lane changing conflicting requests and number of

\begin{tabular}{|c|c|c|}
\hline Investigated Parameter & Symbol & Evaluated Values \\
\hline Cells' reservation buffer time $[\mathrm{s}]$ & $a$ & $1 ; 1.4 ; 1.8 ; 2.2 ; 2.6$ \\
\hline Min. allowed speed to cross reserved cell $[\mathrm{km} / \mathrm{h}]$ & $v_{\beta}$ & $15 ; 17.5 ; 20 ; 22.5 ; 25$ \\
\hline Communication range $[\mathrm{m}]$ & $\mathrm{d}_{\mathrm{cr}}$ & $\mathbf{5 0} ; 75 ; 100 ; 125 ; 150$ \\
\hline Lane-change duration [s] & $\tau$ & $1 ; 1.2 ; 1.4 ; 1.6 ; 1.8$ \\
\hline
\end{tabular}

Table 1 Analyzed control-based parameters

The bold values in Table 1 represent nominal, or default, values for given simulation parameters 
crossing conflicting requests. A conflicting request does not represent a conflict between two trajectories but just denotes a fact that multiple vehicles requested the same block(s) of the time-space domain.

A lane changing conflicting request refers to a situation when a vehicle $i$, which approaches an intersection $z$ and travels in a non-desired lane $\left\{l_{i}(\mathrm{t}): l_{i}(\mathrm{t}) \neq l_{\text {des }, i}(\mathrm{t})\right\}$, is not allowed to change the lane due to the presence of a "conflicting" vehicle $j$ in either adjacent $\left(l_{\text {des }, i}(\mathrm{t})-1\right)$ or desired lane $\left(l_{\mathrm{des}, i}(\mathrm{t})\right)$. The total number of lane changing conflicting requests, across all lanes in the network, is then defined as the number of unique quadruples $(i, j$, $z, l_{\mathrm{i}}$ ).

Similarly, a crossing conflicting request at an intersection $z$ refers to a situation when vehicle $i$ wants to reserve a space block (or cell) $s$ that has already been reserved for another vehicle $\mathrm{j}$ at the requested time $t_{i, s}^{\text {res }}$. The total number of the crossing conflicting requests, across all intersections in the network, is then defined as the number of unique quadruples $(i, j, z, s)$.

\section{Results}

\subsection{Cells' reservation buffer time}

A single cell might be reserved, during distinctive time instances, by vehicles traveling in different directions (see for instance red cell in Fig. 1). To provide safe, yet efficient, control at the intersection it is crucial to provide adequate clearance or safety buffer time, i.e., minimum period of time, between two consecutive vehicle's arrivals, during which a cell is not-occupied. Figure 5 shows how this clearance impacts the efficiency performance measures (left) and surrogate safety indicators (right). The two data series represent mean values from five simulation runs, associated with error bars representing one standard deviation on each side of the mean value. A larger clearance leads to the reduction of intersection capacity that results in an increase in vehicle's delay and number of experienced stops (see Fig. 5 left). The significant degradation in traffic performance (for given traffic demand) is observed at clearance time of $2.2 \mathrm{~s}$ and this might be due to the fact that only one lane is assigned for the through movements (see Fig. 1).

While the larger clearance times undoubtedly result in safer operations within the intersection, it also leads to the higher number of potential intersection and lane changing conflicting requests in the way we interpret these surrogate performance measures (see Fig. 5 right). It is important to note that a larger clearance time leads to a higher number of stops that is often manifested by formation of the vehicular queues. The vehicles waiting in such queues often cause an increased number of lanechanging conflicts when compared to those vehicles that are traveling in the opposite directions; and that want to reach the appropriate lane before the downstream intersection (see the dashed line in Fig. 5 right).

\subsection{Allowed speed to cross the reserved cell}

The reservation cells, as the most valuable space in the traffic network, need to be efficiently utilized by conflicting vehicles so that they are minimally occupied/maximally available. This means that it is our goal for any vehicles to quickly pass over, and clear, the reservation cells so that they become available for other vehicles as soon as possible. We investigate how the minimum defined speed, that can be assigned to the through movement vehicles while traversing through the intersection, impacts the efficiency performance measures and surrogate safety indicators. It is noticeable, from Fig. 6 left, that the minimum allowed speed has significant impact on the vehicle delays and number of stops. Higher speeds lead to the lower delays at the intersections controlled by reservation-based type of control and thereby to the better overall system performances. With higher assigned speeds, the number of crossing conflicting requests, at intersections, is reduced (see Fig. 6 right). This
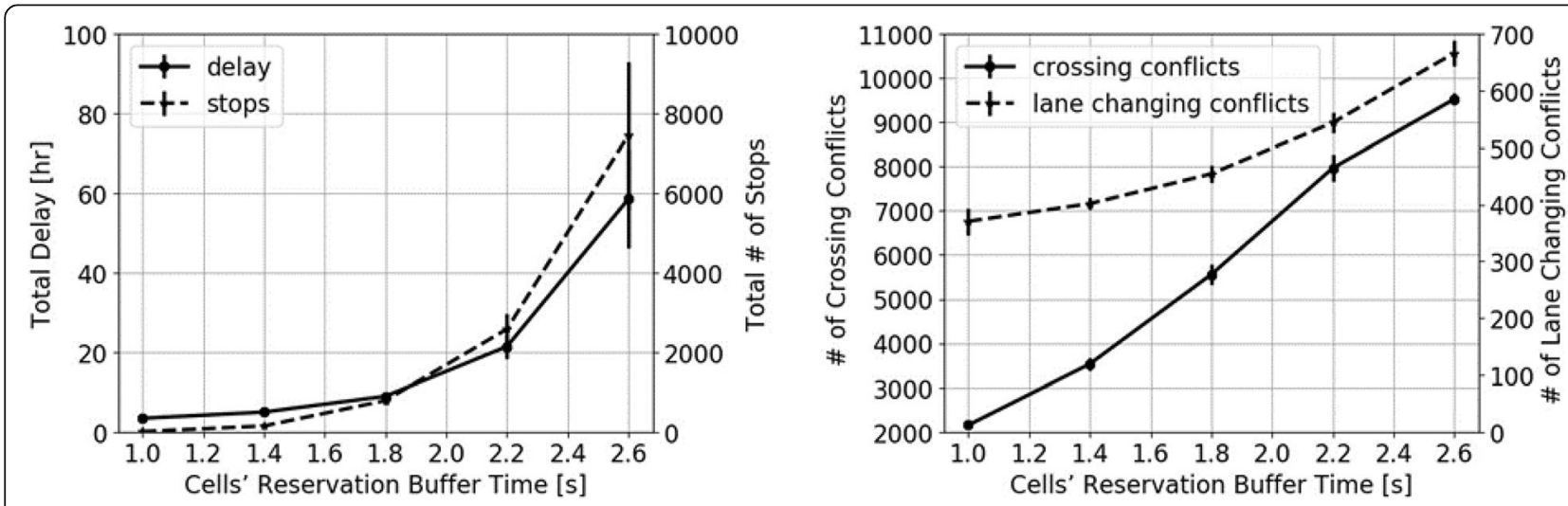

Fig. 5 Impact of reservation buffer time on delays, stops and conflicts 


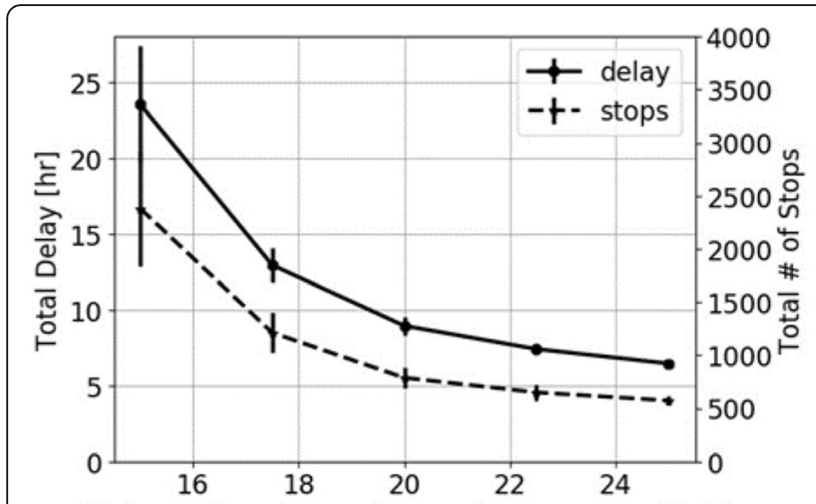

Minimal Allowed Speed through Intersection [kph]

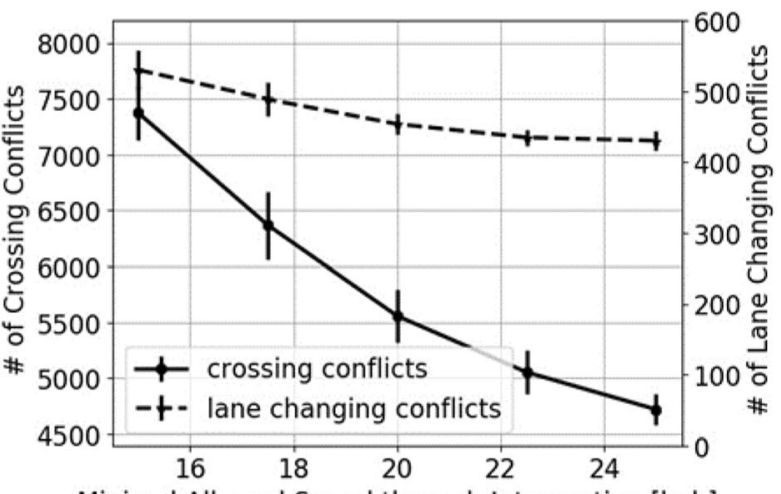

Minimal Allowed Speed through Intersection [kph]

Fig. 6 Impact of cell-crossing speed on delays, stops and conflicts

is a consequence of the reduced time that a vehicle spends within the intersection zone which also reduces a number of competing vehicles that want to cross the intersection at the same time. Similarly, the higher speeds lead to a lower number of stops (see Fig. 6 left) (and shorter queues) that further result in fewer lane changing conflicting requests (see Fig. 6 right).

\subsection{Communication range}

Similar to the applications of Green Light Optimal Speed Advisory (GLOSA) (Stevanovic et al. [19]) we explore here how timely provided information impacts the overall performance of the system. Feedback on the reserved space (and time) at the intersections is provided to a vehicle when it is no more than $50,75,100,125$ and $150 \mathrm{~m}$ from the downstream intersection. Figure 7 left shows a significant drop in delay and number of stops if the communication range is increased from 50 to $75 \mathrm{~m}$. A further increase in communication range distance does not have a significant impact on the efficiency of operations. It is important to note here that the results shown in Fig. 7 left refer to a moderate traffic demand (e.g., LOS C) and they might be different for other traffic demand levels.

Similar results can be observed for the intersection and link based conflicting requests. In fact, the increased number of stops (and potential queues) leads to a higher number of crossing and lane changing conflicting requests, as shown in Fig. 7 right.

\subsection{Lane changing duration}

Figure 8 shows that for a given traffic demand the duration of lane-changing impacts only the number of lanechanging conflicting requests (right) whereas the other efficiency performance measures (left) and surrogate safety indicators remains quite stable (right). As it can be expected, an increase in duration of lane-changing maneuvers often requires more space $(v \cdot \tau)$ for execution of such maneuvers, thereby leading to a higher probability of conflicting requests. It seems that, for a given moderate traffic demand, these conflicting requests "postpone" execution of the lane-changing maneuvers without a significant impact on efficiency (delays and stops) of the traffic, as shown in Fig. 8 left.
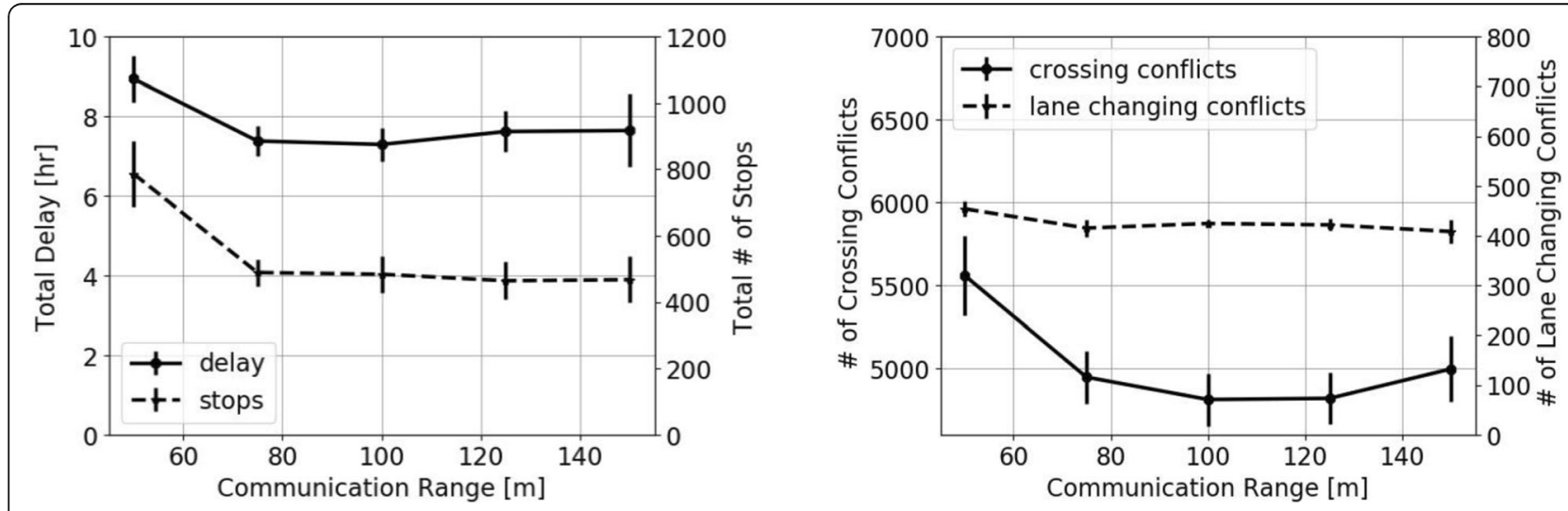

Fig. 7 Impact of communication range on delays, stops and conflicts 

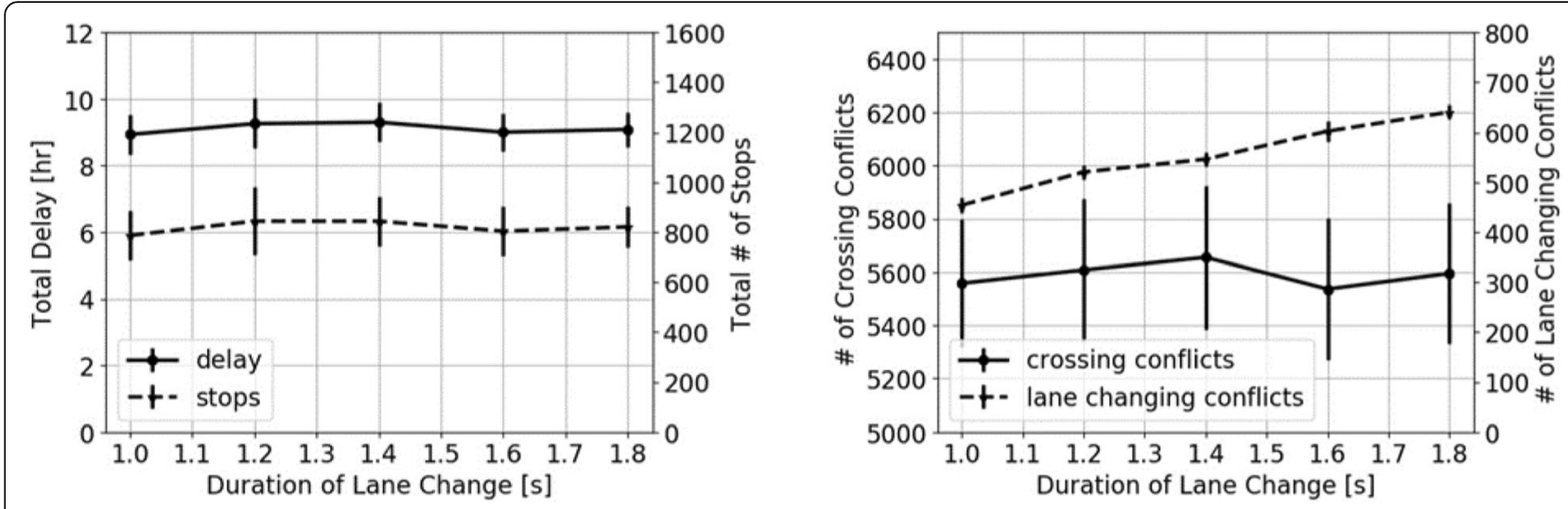

Fig. 8 Impact of lane-changing interval on delay, stops and conflicts

\section{Conclusions}

The goal of this study is to investigate how multiple control-based parameters within CADLARIC (Combined Alternate-Direction Lane Assignment and Reservationbased Intersection Control) impact the efficiency and surrogate safety of autonomous and connected vehicles. The FAUSIM experiments, conducted with moderate traffic demand and multiple random seeds, lead to following conclusions:

- Cells' reservation buffer time, which represents the minimum amount of time between two consecutive vehicular arrivals (at a single cell), has a significant impact on travel delay and number of stops and consequently on conflicting requests. The significant degradation in traffic performance is observed for buffer time of $2.2 \mathrm{~s}$ or larger.

- The minimum allowed speed of through moving vehicles to cross the reserved cells significantly impacts the system performances. Higher speeds tend to lead to lower delays and number of stops at the intersections controlled by reservation-based type of control. The reduced number of stops leads to shorter queues that further result in lower number of conflicting requests at the intersections and mid-blocks.

- An increase in communication range improves efficiency of the system up to certain point, but then the performance stabilizes and further increase in distance (to reserve cells ahead of time) does not significantly impact delays and stops. For the tested scenarios (distances, traffic demand, etc.) this "deflection point" corresponds to the communication range of $75 \mathrm{~m}$.

- Similarly, for the tested traffic demand, the duration of lane-changing process has a significant impact only the number of lane-changing conflicting requests. Longer lane-changing maneuvers often require more space and thus increase probability of having conflicting requests.

Future research should investigate the impact of reservation-based control parameters on the efficiency and surrogate safety indicators for various levels of traffic demand and multiple control strategies. Also, it would be good to investigate how one of the heuristic optimization techniques could be used to find an optimal combination of these parameters to either minimize delays and stops, or conflicting requests, or both.

\section{Abbreviations \\ AIM: Automated intersection management; AV: Automated vehicles; CADLARIC: Combined alternate-direction lane assignment and reservation- based intersection control; CAV: Connected and automated vehicles; FAUSIM: Flexible arterial utilization simulation model; FCFS: First come first serve; GLOSA: Green light optimal speed advisory; LOS: Level of service; OD: Origin-destination; RIC: Reservation-based intersection control}

\section{Acknowledgements}

Not applicable.

\section{Authors' contributions}

The authors confirm contribution to the paper as follows: study conception and design: NM and AS; data collection: NM; analysis and interpretation of results: AS and NM; draft manuscript preparation: AS and NM. All authors reviewed the results and approved the final revised version of the manuscript.

\section{Funding}

The research presented in this paper is not funded by any sponsoring organization.

\section{Availability of data and materials}

The datasets generated and/or analyzed during the current study are not publicly available due intellectual property rights but can be made available from the corresponding author on a reasonable request.

Competing interests

The authors declare that they have no competing interests. 


\section{Author details}

${ }^{1}$ Department of Civil \& Environmental Engineering, Swanson School of Engineering, University of Pittsburgh, 218D Benedum Hall, 3700 O'Hara Street, Pittsburgh, PA 15261, USA. ${ }^{2}$ Siemens Digital ITS Lab, Austin, TX, USA.

Received: 5 July 2019 Accepted: 15 January 2020

Published online: 05 February 2020

\section{References}

1. Ahmane, M., Abbas-Turki, A., Perronnet, F., Wu, J., El Moudni, A., Buisson, J., \& Zeo, R. (2013). Modeling and controlling an isolated urban intersection based on cooperative vehicles. Transportation Research Part C: Emerging Technologies, 28, 44-62.

2. Altché, F., Qian, X., \& de La Fortelle, A. (2016). Time-optimal coordination of mobile robots along specified paths. In 2016 IEEE/RSJ international conference on intelligent robots and systems (IROS) (pp. 5020-5026). IEEE. https://ieeexplore.ieee.org/document/7759737.

3. Choy, M. C., Srinivasan, D., \& Cheu, R. L. (2003). Cooperative, hybrid agent architecture for real-time traffic signal control. IEEE Transactions on Systems, Man, and Cybernetics-Part A: systems and humans, 33(5), 597-607.

4. Dresner, K., \& Stone, P. (2004). Multiagent traffic management: A reservationbased intersection control mechanism. In Proceedings of the third international joint conference on autonomous agents and multiagent systems-volume 2 (pp. 530-537). IEEE Computer Society.https://ieeexplore.ieee.org/document/1373519.

5. Dresner, K., \& Stone, P. (2005). Multiagent traffic management: An improved intersection control mechanism. In Proceedings of the fourth international joint conference on autonomous agents and multiagent systems (pp. 471477). ACM. https://dl.acm.org/doi/10.1145/1082473.1082545.

6. Dresner, K., \& Stone, P. (2008). A multiagent approach to autonomous intersection management. J Artif Intell Res, 31, 591-656.

7. Hassan, A. A., \& Rakha, H. A. (2014). A fully-distributed heuristic algorithm for control of autonomous vehicle movements at isolated intersections. International Journal of Transportation Science and Technology, 3(4), 297-309.

8. Hausknecht, M., Au, T. C., \& Stone, P. (2011). Autonomous intersection management: Multi-intersection optimization. In 2011 IEEE/RSJ international conference on intelligent robots and systems (pp. 4581-4586). IEEE. https:// ieeexplore.ieee.org/document/6094668.

9. HCM 2010. (2010). Highway Capacity Manual. Washington, D.C: Transportation Research Board.

10. Kato, S., Tsugawa, S., Tokuda, K., Matsui, T., \& Fujii, H. (2002). Vehicle control algorithms for cooperative driving with automated vehicles and intervehicle communications. IEEE Trans Intell Transp Syst, 3(3), 155-161.

11. Lee, J., \& Park, B. (2012). Development and evaluation of a cooperative vehicle intersection control algorithm under the connected vehicles environment. IEEE Trans Intell Transp Syst, 13(1), 81-90.

12. Levin, M. W., \& Boyles, S. D. (2015). Intersection auctions and reservationbased control in dynamic traffic assignment. Transp Res Rec, 2497(1), 35-44.

13. Levin, M. W., \& Boyles, S. D. (2016). A cell transmission model for dynamic lane reversal with autonomous vehicles. Transportation Research Part C: Emerging Technologies, 68, 126-143.

14. Levin, M. W., Boyles, S. D., \& Patel, R. (2016). Paradoxes of reservation-based intersection controls in traffic networks. Transp Res A Policy Pract, 90, 14-25.

15. Li, Z., Chitturi, M. V., Zheng, D., Bill, A. R., \& Noyce, D. A. (2013). Modeling reservation-based autonomous intersection control in VISSIM. Transp Res Rec, 2381(1), 81-90.

16. Lin, P., Liu, J., Jin, P. J., \& Ran, B. (2017). Autonomous vehicle-intersection coordination method in a connected vehicle environment. IEEE Intell Transp Syst Mag, 9(4), 37-47.

17. Mitrovic, N., Dakic, l., \& Stevanovic, A. (2019). Combined alternate-direction lane assignment and reservation-based intersection control. In Publication for IEEE Transactions on Intelligent Transportation Systems.

18. Shahriari, M., \& Biglarbegian, M. (2016). A prioritized collision avoidance methodology for autonomous driving. In 2016 IEEE 19th international conference on intelligent transportation systems (ITSC) (pp. 1832-1837). IEEE. https://ieeexplore.ieee.org/document/7795853.

19. Stevanovic, A., Stevanovic, J., \& Kergaye, C. (2013). Green light optimized speed advisory systems: Impact of signal phasing information accuracy. Transp Res Rec, 2390(1), 53-59.

20. Stevanovic, A., \& Mitrovic, N. (2018). Combined alternate-direction lane assignment and reservation-based intersection control. In 21 st international conference on intelligent transportation systems (ITSC) (pp. 14-19). IEEE. https://ieeexplore.ieee.org/document/8569755.

21. Stevanovic, A., \& Mitrovic, N. (2019). Traffic microsimulation for flexible utilization of urban roadways. Transportation Research Record, 2673(10),92104. https://doi.org/10.1177/0361198119848407.

22. Sun, W., Zheng, J., \& Liu, H. X. (2018). A capacity maximization scheme for intersection management with automated vehicles. Transportation Research Part C: Emerging Technologies, Vol 94, (pp. 19-23)

23. Tian, D., Zhu, K., Zhou, J., Wang, J., Zhang, G., \& Liu, H. (2015). A mobility model for connected vehicles induced by the fish school. International Journal of Distributed Sensor Networks, 11(10), 163581.

24. Toledo, T., \& Zohar, D. (2007). Modeling duration of lane changes. Transp Res Rec, 1999(1), 71-78.

25. Vu, H., Aknine, S., \& Ramchurn, S. D. (2018). A decentralised approach to intersection traffic management (pp. 527-533). IJCAl. https://www.ijcai.org/ Proceedings/2018/73.

26. Wageningen-Kessels, F., Van Lint, H., Vuik, K., \& Hoogendoorn, S. (2014). Genealogy of traffic flow models. EURO Journal on Transportation and Logistics, 4(4), 445-473.

27. Wilensky, U. (1999). NetLogo. Center for Connected Learning and Computer-Based Modeling, Northwestern University. Evanston, IL. http://ccl. northwestern.edu/netlogo/..

28. Wu, J., Abbas-Turki, A., \& El Moudni, A. (2012). Cooperative driving: An ant colony system for autonomous intersection management. Appl Intell, 37(2), 207-222.

29. Yang, K., Guler, S. I., \& Menendez, M. (2016). Isolated intersection control for various levels of vehicle technology: Conventional, connected, and automated vehicles. Transportation Research Part C: Emerging Technologies, $72,109-129$.

\section{Publisher's Note}

Springer Nature remains neutral with regard to jurisdictional claims in published maps and institutional affiliations.

\section{Submit your manuscript to a SpringerOpen ${ }^{\circ}$ journal and benefit from:}

- Convenient online submission

- Rigorous peer review

- Open access: articles freely available online

High visibility within the field

- Retaining the copyright to your article

Submit your next manuscript at $\boldsymbol{\nabla}$ springeropen.com 